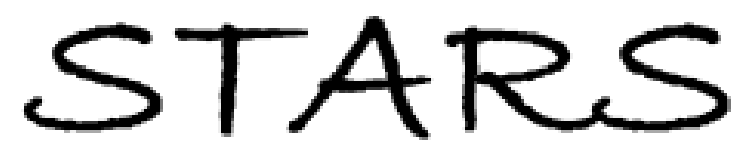

University of Central Florida

STARS

$1-1-2011$

\title{
A Multifunctional Gold Nanoparticle/Polyelectrolyte Fibrous Nanocomposite Prepared from Electrospinning Process
}

Hui Chen

University of Central Florida

Anindarupa Chunder

University of Central Florida

Xiong Liu

University of Central Florida

Feroz Haque

University of Central Florida

Jianhua Zou

University of Central Florida

Find similar works at: https://stars.library.ucf.edu/facultybib2010

University of Central Florida Libraries http://library.ucf.edu

See next page for additional authors

This Article is brought to you for free and open access by the Faculty Bibliography at STARS. It has been accepted for inclusion in Faculty Bibliography 2010 s by an authorized administrator of STARS. For more information, please contactSTARS@ucf.edu.

\section{Recommended Citation}

Chen, Hui; Chunder, Anindarupa; Liu, Xiong; Haque, Feroz; Zou, Jianhua; Austin, Lauren; Knowles, Genevieve; Zhai, Lei; and Huo, Qun, "A Multifunctional Gold Nanoparticle/Polyelectrolyte Fibrous Nanocomposite Prepared from Electrospinning Process" (2011). Faculty Bibliography 2010s. 1159. https://stars.library.ucf.edu/facultybib2010/1159

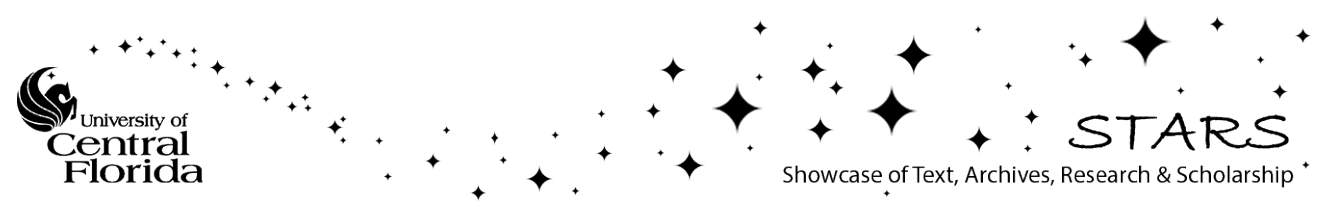




\section{Authors}

Hui Chen, Anindarupa Chunder, Xiong Liu, Feroz Haque, Jianhua Zou, Lauren Austin, Genevieve Knowles, Lei Zhai, and Qun Huo 


\title{
Materials Express
}

\section{A Multifunctional Gold Nanoparticle/ Polyelectrolyte Fibrous Nanocomposite Prepared from Electrospinning Process}

\author{
Hui Chen, Anindarupa Chunder, Xiong Liu, Feroz Haque, Jianhua Zou, Lauren Austin, \\ Genevieve Knowles, Lei Zhai*, and Qun Huo*
}

Department of Mechanical, NanoScience Technology Center, Department of Chemistry and Department of Physics, Materials and Aerospace Engineering, University of Central Florida, 12424 Research Parkway Suite 400,

Orlando, FL, 32826, USA

Gold nanoparticles are introduced to a fibrous nanocomposite material prepared from electrospinning a polyelectrolyte solution of poly(acrylic acid) (PAA) and poly(allylamine hydrochloride) (PAH). The functional groups in the fiber allow convenient thermal crosslinking of the fibers and the binding of tetrachloroaurate ions. Gold nanoparticle-modified nanofibers can be further treated by silver enhancement that increased the electrical conductivity of the nanofibers to $10^{-2} \mathrm{~S} / \mathrm{cm}$. The FT-IR analysis of the nanocomposite fibers shows that the deposition of gold nanoparticles significantly enhances the IR absorption intensity of the polymer fibers, offering a potential sensing capability through enhanced FT-IR absorption of molecules. Upon laser irradiation, the photothemal effect generated by gold nanoparticles caused deformation, melting, or local decomposition of the nanofibers which allows the patterning of nanofibers. The multifunctional composite nanofibers may find many important potential applications in sensors, optical and electronic devices, tissue engineering and catalysis.

Keywords: Electrospun Fibers, Polyelectrolyte, Gold Nanoparticles, Light Responsive.

\footnotetext{
${ }^{*}$ Authors to whom correspondence should be addressed. Emails: qhuo@mail.ucf.edu; lzhai@mail.ucf.edu
}

\section{INTRODUCTION}

Metal nanoparticles such as gold and silver present unique chemical, optical and electrical properties with a wide range of potential applications. The surface plasmon resonance and surface enhanced Raman scattering effect are two optical properties of metal nanoparticles that have been investigated extensively for biological applications. ${ }^{1-7}$ Gold nanoparticles have been found to be capable of catalyzing many electrochemical reactions and processes that are important for sensors, fuel cells, and other industrial applications. ${ }^{8-13}$ Additionally, gold nanoparticles, with their large extinction coefficients of surface plasmon resonance in the visible and near infrared region, are excellent photon-thermal energy converters. This property is currently being explored for the photothermal ablation of tumor tissues, bacteria, and protein aggregates, ${ }^{6,7,14-17}$ and polymer nanocomposite thin films for microstructure fabrication. ${ }^{18}$ Immobilizing gold nanoparticles on various supporting materials including hydrogels, ${ }^{19,20}$ porous silica, ${ }^{21,22}$ polymer thin films ${ }^{23}$ and polymer fibers ${ }^{13}$ has been extensive investigated to extend the applications of gold nanoparticles. Among these studies, one of the goals is to achieve large surface area of the supporting materials to improve the utilization of gold nanoparticles. Polymer nanofibers are very attractive supporting materials for gold nanoparticles due to their tunable chemical properties and large surface area.

Among various methods of fabricating polymeric fibers, electrospinning has become a popular method to generate continuous, ultrathin fibers with diameters on the order of microns and sub-microns from a variety of polymeric materials. ${ }^{24}$ In a typical electrospinning process, a high 
electrostatic field is applied to a polymer solution held in a syringe with a needle. A jet is emitted from the conelike meniscus (Tayler cone) formed on the needle when the field strength excesses the critical value to overcome the polymer solution surface tension. As the jet moves toward the collecting electrode, its diameter decreases due to lateral excursions, and the solvent evaporates, leading to a non-woven fabric mat on the collecting electrode. Electrospun nanofibers possess important properties including high surface area, small fiber diameter, high permeability, and the potential to incorporate active chemistry. These properties make the electrospun fiber mats promising candidates for extensive applications including drug delivery, filtration, catalysis, sensing, composite applications, and tissue engineering. ${ }^{25-29}$ The combination of the unique chemical, optical and electrical properties of metal nanoparticles with the high surface area provided by polymer nanofibers has lead to further advanced and multifunctional nanocomposite materials with many promising applications in these areas. To name a few, poly(methyl methacrylate) (PMMA) electrospun fibers was coated with poly(aniline) (PANi) to host gold nanoparticles and used as matrix for enzymes such as superoxide dismutase (SOD) for the applications as superoxide sensors. ${ }^{13}$ Gold nanoparticles were loaded in electrospun polyethyleneimine (PEI)/polyvinylalcohol (PVA) fibers for catalytic applications. ${ }^{30}$ While polymer electrospun fibers offer promising templates to host gold nanoparticles, functional groups are required for gold nanoparticle attachment and crosslinking is needed to obtain stable polymer fibers which usually involves extra chemical treatment such as the exposure to glutaraldehyde (GA) vapor. Therefore,

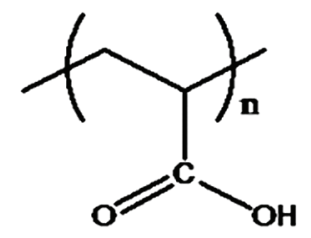

PAA

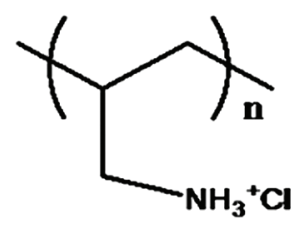

a

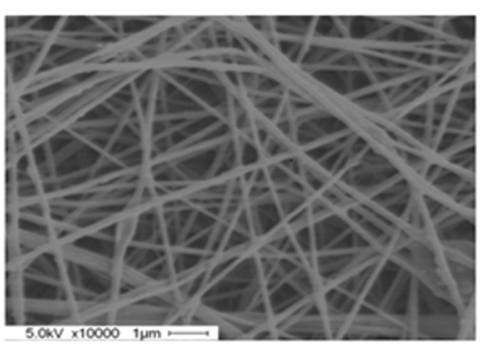

PAH

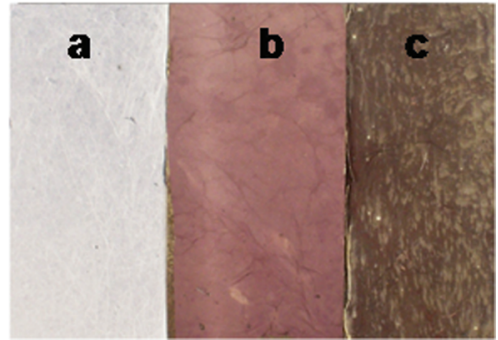

Fig. 1. The chemical structures of poly(acrylic acid) (PAA) and poly(allylamine hydrochloride) (PAH); SEM image of pure fibers (upperright) and digital images (bottom-right) of pure fiber mats (a), fiber mats after the gold nanoparticle deposition (b) and after further treatment of silver enhancement (c). a polymer fiber system that has functional groups to attach gold nanoparticle and can be crosslinked without extra chemical treatment would provide a cost effective approach to host gold nanoparticles.

Recently, Chunder et al. reported a convenient method to fabricate polyelectrolyte electrospun fibers comprising of two polyelectrolytes with opposite charges, poly(acrylic acid) (PAA) and poly(allylamine hydrochloride) (PAH) (Fig. 1). ${ }^{31}$ These polyelectrolytes provide different functional groups (carboxylate and amine) to bind metal ions for the nanoparticle fabrication and a simple heat treatment can crosslink the fibers through the formation of amide bond between amine and carboxylate groups. Such interesting propoerties make PAA/PAH electrospun fibers unique system to support gold nanoparticles. In this paper, we report the deposition of gold nanoparticles onto PAA/PAH electrospun fibers and the investigation of various properties including FT-IR enhancement and photothermal effect upon laser irradiation.

\section{EXPERIMENTAL DETAILS}

\subsection{Preparation of Nanocomposite Fibers}

An aqueous solution of polyelectrolyte was made by mixing 0.668 g poly(acrylic acid) (PAA, 25\% aqueous solution, Mw 90000, Polysciences, Inc.) and $0.083 \mathrm{~g}$ poly(allylamine hydrochloride) (PAH, MW 70000, SigmaAldrich, Inc.) to fabricate the nanofiber according to a reported procedure. ${ }^{31}$ The solution was then loaded in a plastic syringe with a blunt stainless steel needle (22 gauge) and fed constantly by a syringe pump at $0.01 \mathrm{~mL} / \mathrm{h}$. Electrospun fibers was obtained by applying a voltage of $10-15 \mathrm{kV}$ with a high voltage DC power supply (Glassman High Voltage, Inc). A plastic substrate with an indium tin oxide conductive coating (MIL-PRF-131J from Ludlow Coated Product, Inc.) was used to collect the fibers. Two methods were used to introduce gold nanoparticles into the fibers:

Method A: PAA/PAH nanofiber was fabricated and the fiber mat was then heated to $140{ }^{\circ} \mathrm{C}$ overnight to crosslink the fibers. Gold nanoparticles were generated by first dipping the fibers into a $0.01 \mathrm{M}$ chloroauric acid aqueous solution $\left(\mathrm{HAuCl}_{4} \cdot \mathrm{H}_{2} \mathrm{O}\right.$, Sigma-Aldrich, Inc.) and then to a $0.1 \mathrm{M}$ reducing reagent solution (sodium borohydride, Sigma-Aldrich, Inc.). The fiber was washed extensively with deionized water after each dipping to eliminate loosely adsorbed chloroauric acid and reducing reagent. After gold nanoparticle loading, the fiber mats were dried in a desiccator overnight.

Method B: An appropriate amount of polyelectrolyte, $\mathrm{PAA}$ and $\mathrm{PAH}$, with $10 \mathrm{wt} \%$ (with respect to weight of polymers) of chloroauric acid was dissolved in a cosolvent ( $0.13 \mathrm{~g}$ of $N, N$-dimethylformamide and $0.15 \mathrm{~g}$ of deionized water) to make a homogenous solution for electrospinning. A blunt platinum needle (Hamilton Company) 
with a 22 gauge was used to prepare the fibers. The fiber was then heated to $140{ }^{\circ} \mathrm{C}$ overnight. The color of the fibers turned into pink reddish after the thermal treatment.

\subsection{Silver Enhancement}

Silver enhancer kit was purchased from Sigma-Aldrich, Inc. The solution A (silver salt) was mixed with an equal volume of solution B (initiator) just prior to use. The fiber mats with gold nanoparticles prepared from method B were immersed into the silver enhancement solution for 8 minutes. After washing with copious amount of deionized water, the silver enhancer treated fiber mats were dipped in a $2.5 \%$ sodium thiosulfate solution for 2 minutes to stablize the silver coating. The resulting fiber mats were dried in a desiccator overnight.

\subsection{Characterization}

Transmission electron microscopy (TEM) analysis: Electrospun fibers were directly deposited onto TEM copper grids and examined by a FEI Tecnai F30 TEM.

Scanning electron microscope (SEM): The fibers on the conductive plastic substrate were used directly for SEM study. In order to avoid electric charge build-up, the fibers were coated with a Pd-Au film by an Emitech Magnetron Sputter Coater before imaging. The specimens then were observed by a JEOL 6400 SEM at an accelerating voltage of $5 \mathrm{kV}$.

Conductivity measurement: Three types of nanofibers, pure PAA/PAH polyelectrolyte fibers, fibers loaded with gold nanoparticles by method B and the fibers (preloaded with method A) followed by silver enhancement treatment, were released from conductive plastic substrates by immersing in 4\% KOH solution for 2-3 minute. After rinsing with copious deionized water, the free-standing mats were transferred onto glass cover slides. The resulting fiber coatings were dried in a desiccator overnight. The electrodes were then prepared by applying two strips of silver paint on the fiber mats. The distance between two electrodes was $0.15 \mathrm{~cm}$ and the length was $0.6 \mathrm{~cm}$. The average thickness of the fiber mat was measured by an Alpha Step Profilometer. The conductivity was tested by two probe method with a Keithley 6517A Electrometer/HighResistance meter. The conductivity was calculated from the equation: $\sigma=l / R A$, where $R$ is the measured resistance, $A$ is the area (the thickness of the film $\times$ the length of the electrode), and $l$ is the distance between two electrodes. For each type of fiber mats, the conductivity was collected on three different positions. The final reported conductivity was obtained by averaging three measurement results.

ATR-FTIR measurement: The fiber mat was pressed on the surface of a diamond crystal sample plate by an attached pressure arm and the spectra were collected with a
PerkinElmer Spectrum ${ }^{\mathrm{TM}} 100$ FT-IR spectrometer equipped with a universal ATR sampling accessory.

\section{RESULTS AND DISCUSSION}

In this study, two methods were used to incorporate gold nanoparticles into the electrospun PAA/PAH nanofibers. The first one (Method A) involves the deposition of tetrachloroaurate ions $\left(\mathrm{AuCl}_{4}{ }^{-}\right)$onto nanofibers followed by the reduction using sodium borohydride $\left(\mathrm{NaBH}_{4}\right)$. After the electrospun PAA/PAH fibers were deposited onto a conductive plastic substrate, the resulting fabric was thermally crosslinked at $140{ }^{\circ} \mathrm{C}$ overnight to to withstand high ionic strength solutions. ${ }^{24}$ The crosslinked fibers were then immersed in a chloroauric acid solution $\left(\mathrm{HAuCl}_{4}\right)$ in which tetrachloroaurate ions were adsorbed onto the nanofiber surface through ionic interactions. After the immersion, copious water was used to wash off any loosely adsorbed chemicals. The subsequent treatment of the gold ion loaded fibers with a reducing agent $\left(\mathrm{NaBH}_{4}\right)$ solution generated gold nanoparticles on the surface of the prefabricated PAA/PAH fibers. As shown in the TEM micrographs (Figs. 2(a and b)), gold nanoparticles were clearly formed

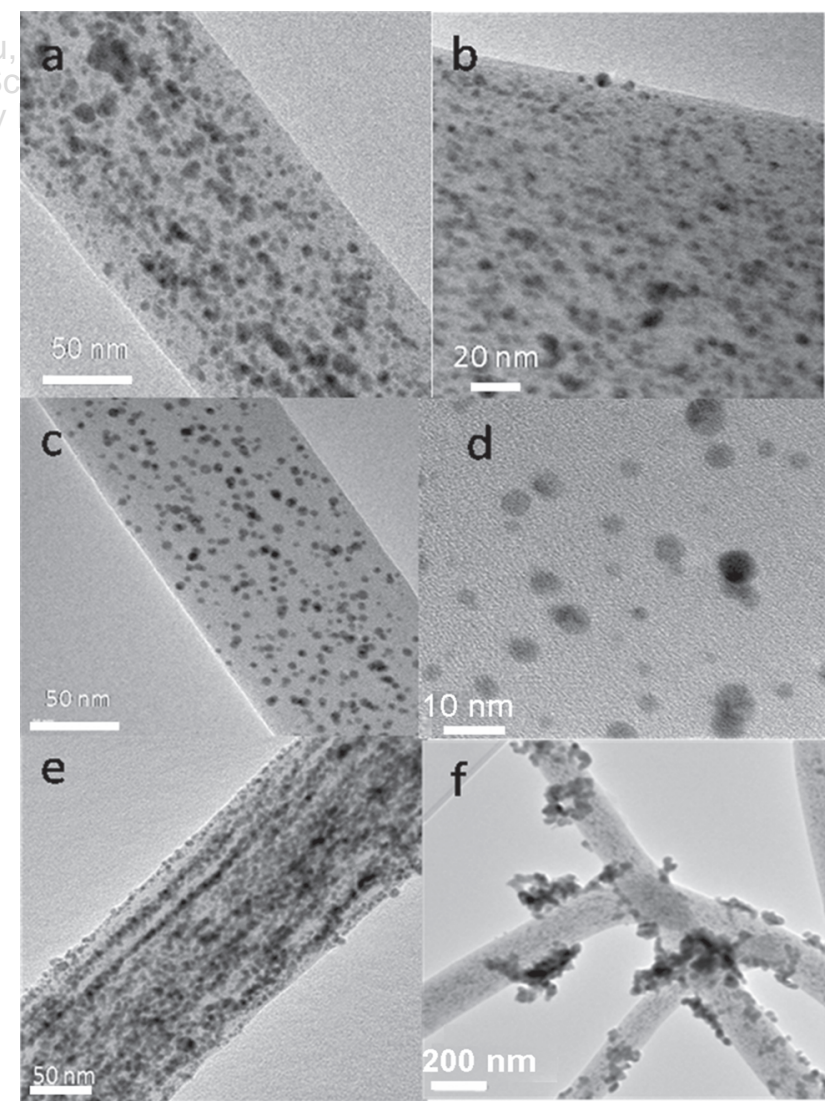

Fig. 2. TEM images of electrospun nanofibers after the gold nanoparticle deposition using method A ( $\mathrm{a}$ and $\mathrm{b}$ ); method B (c and d); method A followed by method B (e); and nanocomposite fibers prepared using method A after further silver enhancement (f). 
on the nanofibers. The average diameter of the nanoparticles is around $8 \mathrm{~nm}$. By applying multiple immersion cycles in $\mathrm{HAuCl}_{4}$ and $\mathrm{NaBH}_{4}$ solutions, the concentration of gold nanoparticles immobilized on the nanofiber surface can be increased accordingly. The nanoparticle-modified fiber mat appeared in a pink-reddish color (Fig. 1).

In a second method (Method B), gold nanoparticles were generated in nanofibers by an amine reduction. It has been reported that long chain aliphatic amine and polymers containging amine groups can be used to reduce gold salts and form stable gold nanoparticles at elevated temperature. ${ }^{32-36}$ Since one of the components in polyelectrolyte solution, PAH, contains free amine groups, we hypothesized that if chloroauric acid was directly mixed to the polyelectrolyte solution, gold nanoparticles may be generated in-situ by a thermal treatment of the electrospun nanofibers. A polyelectrolyte solution with chloroauric acid was successfully electrospun into nanofibers with the same average diameter and morphology as pure polymer nanofibers. In order to prevent the corrosion of the metal needle by chloroauric acid at high voltage, a platinum syringe needle was used for the electrospinning. The electrospun fibers were then heated over $140{ }^{\circ} \mathrm{C}$ for 4 hours. Without any additional reducing agent, gold nanoparticles formed inside the fiber as revealed by the TEM images (Figs. 2(c and d)). The average diameter of gold nanoparticles generated from this method is around 5-8 $\mathrm{nm}$.

Compared with Method A, Method B can generate nanoparticles with more uniform size distribution and less aggreation of the particles. The homogenous mixture of a chloroauric acid solution with PAA/PAH solution allows the uniform distribution of particles in fibers. On the other hand, the nanoparticle distribution in the composite fibers fabrication through Method A shows a gradient with a crease number of nanoparticles towards the fiber core. Such gradient may be due to the rinse of $\mathrm{AuCl}_{4}{ }^{-}$ions by water which tends to remove the $\mathrm{AuCl}_{4}{ }^{-}$ions close to fiber surfaces. Therefore, Method B provide a better control of the nanoparticle size and distribution in the fibers. The gold nanoparticle content in the fibers can be further increased by multiple cycles of immersion of the gold nanoparticle-loaded fibers in a $\mathrm{HAuCl}_{4}$ solution and a sodium borohydride solution (Fig. 2(e)).

Gold nanoparticles and colloids can catalyze the reduction of silver ions into silver islands and colloids through a process called silver enhancement. ${ }^{37}$ In this study, we applied the silver enhancement to the gold nanoparticlemodified nanofibers to generate silver colloids. As shown in Figure 1, after silver enhancement, the color of the nanofiber mat changed from reddish pink to light brown. A TEM micrograph (Fig. 2(f)) reveals the formation of silver nanoparticles and aggregated silver islands on the fiber. Gold and silver nanostructures are known to exhibit surface-enhanced Raman scattering property, which holds promises for biomolecular detection and chemical sensing.
By combining with the high surface area of nanofibers, the composite nanofibers reported here may provide a suitable material for this application.

The FT-IR spectroscopy studies of gold nanoparticleloaded nanocomposite fibers revealed some very interesting IR absorption enhancement effect of gold nanoparticles to the polymer fiber. In the FT-IR studies, the nanofiber mats were pressed onto the diamond plate of an ATR sampling accessory of the FT-IR spectrometer using an attached pressure arm. Compared to pure polymer fibers, the IR absorption intensity of the nanofiber after the gold nanoparticle formation increased dramatically (Fig. 3), particularly the broad band around $3300 \mathrm{~cm}^{-1}$ and the carboxylate stretching band at $1550 \mathrm{~cm}^{-1}$ attributed to the carbonyl groups in PAA. ${ }^{30}$ Because the ATR technique used here for the nanofiber analysis is a surfacesensitive technique and the nanofiber sample mat is not a homogeneous sample, a quantitative conclusion on the enhancement effect could not be obtained from this study. However, the comparison studies of multiple nanofiber samples revealed that the absorption intensity from gold nanoparticle-modified nanofibers was always the strongest. In average, the absorbance of the carboxylate stretching band at $1550 \mathrm{~cm}^{-1}$ is $4-5$ times stronger for nanocomposite fibers than that of pure polymer fibers. The absorption at $3300 \mathrm{~cm}^{-1}$ is attributed to water that remained in the fiber after gold nanoparticle loading. The surface-enhanced IR absorption (SEIRA) effect of metal nanoparticles has been reported previously, though not very extensively. ${ }^{38-41}$ The enhancement mechanism was attributed to similar effect as the surface enhanced Raman scattering (SERS), presumably due to both electromagnetic coupling and chemical effect. While investigation is required to futher understand the impact of gold nanoparticles to the IR abosroption of hosting systems, the SEIRA effect makes the nanocomposite fibers potentially useful materials for molecular sensing applications.

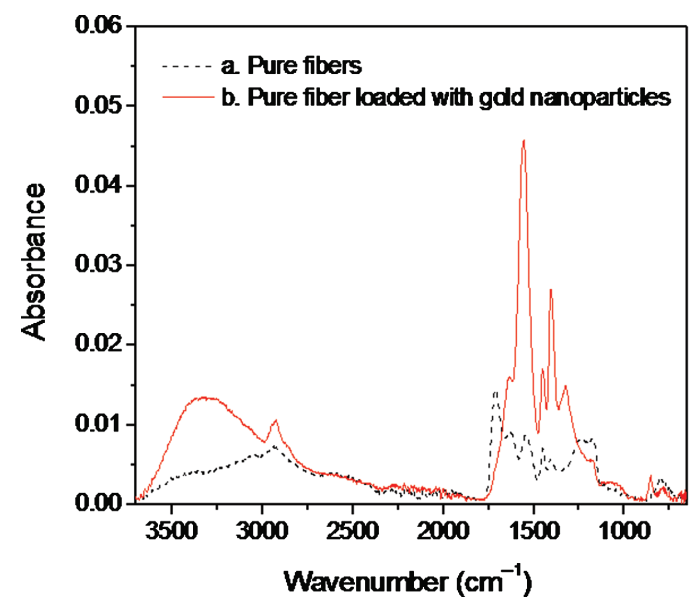

Fig. 3. FTIR spectra of (a) electrospun pure polymer fibers; (b) electrospun composite fibers with gold nanoparticles formed using method A after gold salt immersion-reduction treatment with 6 cycles. 
One of the most appealing potential applications of electrospun nanofibers is as a scaffold material for tissue engineering. ${ }^{26}$ It has been known that many of the cell functions, such as surface adhesion, proliferation, migration and differentiation, can be modulated by electrical stimulation. Nanofiber scaffolds have been prepared from conducting polymers such as polyaniline (PANi) and studied extensively for cell adhesion and proliferation. ${ }^{42}$ The electrical conductivity of the nanocomposite fibers was also investigated in our study. The measured volume conductivity of pure polymer fiber, gold nanoparticle-modified fiber, and fibers further treated by silver enhancement is in the order of $10^{-9}, 10^{-7}$, and $10^{-2} \mathrm{~S} / \mathrm{cm}$, respectively. The silver-enhancement treated gold nanoparticle/PAA/PAH nanofiber has the conductivity in the same range as a PANi nanofiber used in the work by Lelkes et al. on the study of an $\mathrm{H} 9 \mathrm{c} 2$ rat cardiac myoblast cell growth. ${ }^{42}$ This level of conductivity is considered as adequate for studying the effect of electrical stimulation on cell growth and differentiation in vitro.

Another important property of the gold nanoparticlemodified nanofibers is the photothermal effect upon laser irradiation. The extinction coefficients of gold nanoparticles are orders of magnitude higher than typical organic dye molecules. ${ }^{43}$ Gold nanoparticles can convert the

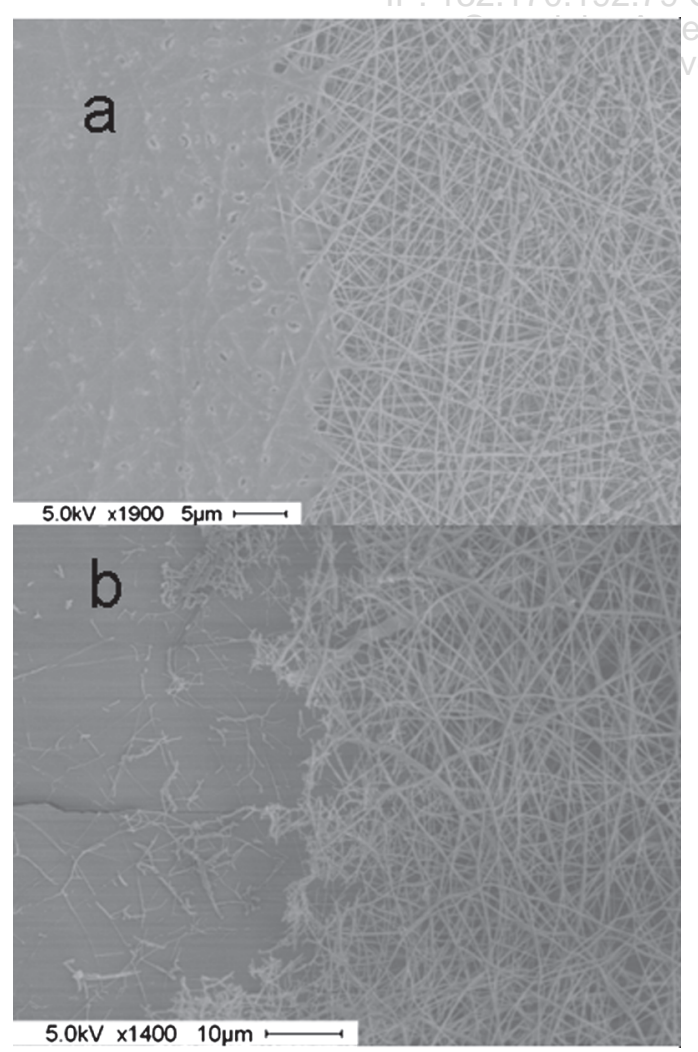

Fig. 4. SEM images of laser-irradiated composite nanofibers using a $532 \mathrm{~nm}$ CW Nd:YAG laser at $100 \mathrm{~mW}$ for 2 minutes. Gold nanoparticles were generated on the nanofibers using method A after a 3-cycle (a) and 6-cycle (b) of gold salt immersion-reduction process. photon energy to thermal energy efficiently upon laser irradiation. ${ }^{18,44,45}$ The photothermal response of gold nanoparticle-modified nanofibers was studied. The electrospun nanofibers after three cycles of treatment with a $\mathrm{HAuCl}_{4}$ solution followed by a sodium borohydride reduction were irradiated by a $532 \mathrm{~nm} \mathrm{CW} \mathrm{Nd:YAG} \mathrm{laser} \mathrm{at}$ $100 \mathrm{~mW}$ for 2 minutes. The fibers melted under the irradiation as shown in Figure 4(a). Complete decomposition of the fibers was observed when the fiber was treated with six cycles of nanoparticle formation process (Fig. 4(b)). The photothermal effect and deformation of the nanofiber can be adjusted by tuning the nanoparticle concentration as well as the laser power and irradiation time. Such property may lead to the application of the composite nanofibers as light responsive scaffold materials for tissue engineering studies. For example, a patterned nanofiber scaffold can be prepared by the laser irradtion through a photomask which has potential applications in evaluating cell behavior with and without nanofiber scaffolds.

\section{CONCLUSIONS}

In summary, we generated gold nanoparticles in PAA/PAH polymer electrospun nanofibers with controlled nanoparticle content. The functional groups in the fibers offer convenient binding with metal ions and allow for simple thermal crosslinking of the fibers and the reduction gold ions, offering a cost effective approch to host gold nanoparticles. The resulting nanofibrous composite materials demonstrate multifunctional properties including high electrical conductivity, photothermal response, surface-enhanced IR absorption. These materials may find many potential applications in the areas of sensor, optical and electronic devices, tissue engineering and catalysis.

Acknowledgment: This work is supported by the National Science Foundation CAREER award DMR 0552295, NIRT award CMMI 0506531, and NUE award EEC-0741508.

\section{References and Notes}

1. D. A. Schultz, Curr. Opin. Biotechnol. 14, 13 (2003).

2. M.-C. Daniel and D. Astruc, Chem. Rev. 104, 293 (2004)

3. G. Schmid, Clusters and Colloids: From Theory to Applications, $\mathrm{VCH}, \mathrm{New}$ York (2004).

4. Q. Huo, Colloids Surf. B-Biointerfaces 59, 1 (2007).

5. I. H. El-Sayed, X. Huang, and M. A. El-Sayed, Nano Lett. 5, 829 (2005).

6. X. Huang, I. H. El-Sayed, W. Qian, and M. A. El-Sayed, J. Am. Chem. Soc. 128, 2115 (2006).

7. C. Loo, A. Lowery, N. Halas, J. West, and R. Drezek, Nano Lett. 5, 709 (2005).

8. J. Das, A. M. Abdul, and H. Yang, J. Am. Chem. Soc. 128, 16022 (2006).

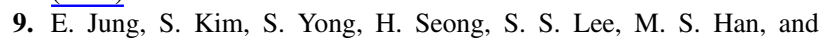
S. Lee, Angew. Chem. Int. Ed. 50, 4386 (2011).

10. J. Deng, W. Shih, and C. Mou, J. Phys. Chem. C 111, 9723 (2007). 
11. V. Mazumder, Y. Lee, and S. Sun, Adv. Funct. Mater. 20, 1224 (2010).

12. K. Matsuoka, K. Miyazaki, Y. Iriyama, K. Kikuchi, T. Abe, and Z. Ogumi, J. Phys. Chem. C 111, 3171 (2007).

13. P. Santhosh, K. M. Manesh, S.-H. Lee, S. Uthayakumar, A. I. Gopalan, and K.-P. Lee, Analyst 136, 1557 (2011).

14. D. P. O'Neal, L. R. Hirsch, N. J. Halas, J. D. Payne, and J. L. West, Cancer Lett. 209, 171 (2004).

15. D. Pissuwan, S. M. Valenzuela, and M. B. Cortie, Trends Biotechnol. 24, 62 (2006).

16. V. P. Zharov, K. E. Mercer, E. N. Galitovskaya, and M. S. Smeltzer, Biophys. J. 90, 619 (2006).

17. M. J. Kogan, N. G. Bastus, R. Amigo, D. Grillo-Bosch, E. Araya, A. Turiel, A. Labarta, E. Giralt, and V. F. Puntes, Nano Lett. 6, 110 (2006).

18. H. Chen, X. Liu, H. Muthuraman, J. Zou, Q. Dai, J. Wang, and Q. Huo, Adv. Mater. 18, 2876 (2006).

19. A. Skardal, J. Zhang, L. McCoard, S. Oottamasathien, and G. D. Prestwich, Adv. Mater. 22, 4736 (2010).

20. X. Zhao, X. Ding, Z. Deng, Z. Zheng, Y. Peng, and X. Long, Macromol. Rapid Commun. 26, 1784 (2005)

21. H. Zhu, B. Lee, S. Dai, and S. H. Oberbury, Langmuir 19, 3974 (2003).

22. R. I. Nooney, T. Dhanasekaran, Y. Chen, and R. J osephs, Adv. Mater. 14, 529 (2002).

23. K. Akamatsu and S. Deki, J. Mater. Chem. 7, 1773 (1997).

24. J. Lyons and F. K Ko, Ency. Nanosci. Nanotechnol. 6, 727 (2004).

25. N. Ashammakhi, A. Ndreu, A. M. Piras, L. Nikkola, T. Sindelar, H. Ylikauppila, A. Harlin, M. E. Gomes, N. M. Neves, E. Chiellini, F. Chiellini, V. Hasirci, H. Redl, and R. L. Reis, J. Nanosci. Nanotechnol. 7, 862 (2007).

26. R. Murugan and S. Ramakrishna, Tissue Engin. 12, 435 (2006).

27. J. T. McCann, D. Li, and Y. Xia, J. Mater. Chem. 15, 735 $\underline{\text { (2005). }}$
28. G.-M. Kim, A. Wutzler, H.-J. Radusch, G. H. Michler, P. Simon, R. A. Sperling, and W. J. Parak, Chem. Mater. 17, 4949 (2005).

29. K. Mueller, J. F. Quinn, A. P. R. Johnston, M. Becker, A. Greiner, and F. Caruso, Chem. Mater. 18, 2397 (2006).

30. X. Fang, H. Ma, S. Xiao, M. Shen, R. Guo, X. Cao, and X. Shi, J. Mater. Chem. 21, 4493 (2011)

31. A. Chunder, S. Sarkar, Y. Yu, and L. Zhai, Colloid. Surf., B: Biointerfaces 58, 172 (2007).

32. X. Liu, J. Wang, M. Atwater, Q. Dai, J. Zou, J. P. Brennan, and Q. Huo, J. Nanosci. Nanotechnol. 7, 3126 (2007).

33. H. Hiramatsu and F. E. Osterloh, Chem. Mater. 16, 2509 (2004).

34. M. Aslam, L. Fu, M. Su, K. Vijayamohanan, and V. P. Dravid, J. Mater. Chem. 14, 1795. (2004).

35. A. Rai, A. Prabhune, and C. C. Perry, J. Mater. Chem. 20, 6789 (2010).

36. M. A. Nash, J. J. Lai, A. S. Hoffman, P. Yager, and P. S. Stayton, Nano Lett. 10, 85 (2010).

37. K. Bienz, D. Egger, and L. Pasamontes, J. Histochem. Cytochem. 34, 1337 (1986).

38. D. Enders, Surface Enhanced Infrared Absorption on Au Nanoparticle Films For Optical Biosensing, Dissertation, Ruperto-Carola University of Heidelberg, Germany (2005).

39. C. Domingo, V. Resta, S. Sanchez-Cortes, J. V. Garcia-Ramos, and J. Gonzalo, J. Phys. Chem. C 111, 8149 (2007).

40. D. Enders and A. Pucci, Appl. Phys. Lett. 88, 184104/1 (2006).

41. D. Enders, T. Nagao, T. Nakayama, and M. Aono, Langmuir 23, 6119 (2007).

42. M. Li, Y. Guo, Y. Wei, A. G. MacDiarmid, and P. I. Lelkes, Biomater. 27, 2705 (2006).

43. Z.-S. Wu, H. Lu, X. Liu, R. Hu, H. Zhou, G. Shen, and R.-Q. Ru, Anal. Chem. 82, 3890 (2010).

44. K. Vanherck, S. Hermans, T. Verbiest, and I. Vankelecom, J. Mater. Chem. 21, 6079 (2011).

45. S. Maity, L. N. Downen, J. R. Bochinski, and L. I. Clarke, Polymer 52, 1674 (2011).

Received: 6 April 2011. Revised/Accepted: 31 May 2011. 